\title{
Comparação entre duas dietas à base de leite humano em relação ao crescimento e à mineralização óssea de recém-nascidos de muito baixo peso
}

\author{
Comparison of two diets with modified human milk regarding growth and bone mineralization of very \\ low birth weight infants
}

Ana Beatriz Gonçalves ${ }^{1}$, Salim Moisés Jorge ${ }^{2}$, Arthur Lopes Gonçalves ${ }^{2}$

\section{RESUMO}

Objetivo: Comparar a eficácia e tolerabilidade de duas dietas à base de leite humano $(\mathrm{LH})$ acrescido de fórmula láctea $\left(\right.$ PreNan $^{\circledR}$ ) ou complemento nutricional especial $\left(\right.$ FM $85^{\circledR}$ ) na promoção do crescimento pôndero-estatural, mineralização óssea e tempo de hospitalização de recém-nascidos de muito baixo peso (RNMBP).

Método: Foram constituídos, por sorteio, dois grupos de crianças acompanhadas a partir do $15^{\circ}$ dia de vida, até atingir o peso de alta (2000 $\pm 20 \mathrm{~g})$ : Grupo A, 14 RNMBP receberam $\mathrm{LH}+\mathrm{FM} 85^{\circledR}(5 \mathrm{~g} / 100 \mathrm{~mL} \mathrm{LH})$; Grupo B, 11 RNMBP receberam $\mathrm{LH}+\operatorname{PreNan}^{\circledR} 19 \%$ em volumes iguais. Foram avaliados: peso, comprimento, perímetro cefálico e prega cutânea tricipital média esquerda, calculando-se os incrementos de peso e o tempo para atingir 2000g. Foram dosados: cálcio, fósforo, magnésio e creatinina séricos e urinários e fosfatase alcalina sérica, calculando-se as taxas de reabsorção tubular de fósforo (\%TPR). A mineralização óssea foi avaliada por meio de técnicas radiológicas padronizadas.

Resultados: 11 RNMBP de cada grupo completaram o estudo. Ambas as dietas foram bem toleradas e os índices antropométricos e dosagens séricas iniciais não apresentaram diferenças entre os grupos. Os incrementos de peso do Grupo B foram superiores aos do Grupo A e a fosfatase alcalina sérica do Grupo A foi maior que do Grupo B no final da observação. Não houve diferenças entre os grupos quanto à \% TPR; mineralização óssea e tempo de hospitalização.

Conclusões: Ambas as dietas foram bem toleradas. O leite humano enriquecido com PreNan ${ }^{\circledR} 19 \%$ volume a volume $(v / v)$ se mostrou mais eficiente em relação ao ganho de peso e ao metabolismo ósseo em comparação ao leite humano acrescido de FM $85^{\circledR}$.

Palavras-chave: nutrição; mineralização óssea; crescimento; recém-nascido; prematuro.

\section{ABSTRACT}

Objective: To compare the efficacy and tolerability of two diets based on human milk (HM) added with PreNan ${ }^{\circledR}$ or FM $85^{\circledR}$ on physical growth, bone mineralization and duration of hospitalization.

Methods: Two groups of very low birth weight (VLBW) infants were studied: Group A, 14 infants fed with $\mathrm{HM}+\mathrm{FM} 85^{\circledR}(5 \mathrm{~g} / 100 \mathrm{~mL} \mathrm{HM})$ and Group B, 11 infants fed with $\mathrm{HM}+\operatorname{PreNan}^{\circledR} 19 \%$ volume to volume (v/v), from the $15^{\text {th }}$ day of life up to $2000 \mathrm{~g}$ of weight. The parameters measured were: weight, length, head circumference; mean left triciptal skinfold thickness; calcium, phosphorus, magnesium and creatinine (serum and urine) and alkaline phosphatase (serum) at the beginning and at the end of the observation. The tubular phosphorus reabsorption rate (\% TPR) was calculated and bone mineralization was assessed by X-ray.

Results: 11 infants of each group completed the study. There were no differences between the two groups at the beginning of the study. Weight gain per day $(\mathrm{g} / \mathrm{d})$ or per $\mathrm{kg}$ per day $(\mathrm{g} / \mathrm{kg} / \mathrm{d})$ was significantly higher in Group B, and duration of hospitalization was shorter. Serum calcium, phosphorus and magnesium levels did not differ between groups, but alkaline phosphatase levels were higher in Group $A$ at the end of the study. Groups were similar regarding bone mineralization and \% TPR.
Instituição: Hospital das Clínicas da Faculdade de Medicina de Ribeirão Preto da Universidade de São Paulo (USP), Ribeirão Preto, SP, Brasil

${ }^{1}$ Mestre em Pediatria pela Faculdade de Medicina de Ribeirão Preto da USP, Ribeirão Preto, SP, Brasil

2Professor titular do Departamento de Puericultura e Pediatria da Faculdade de Medicina de Ribeirão Preto da USP, Ribeirão Preto, SP, Brasil
Endereço para correspondência:

Ana Beatriz Gonçalves

Rua Carlos Rateb Cury, 697, Casa 82

CEP 14110-000 - Ribeirão Preto/SP

E-mail: abgoncal@hotmail.com

Recebido em: 6/10/08

Aprovado em: 8/5/09 
Conclusions: Both diets were well tolerated. In terms of weight gain and bone metabolism, supplementation of HM with $\operatorname{PreNan}{ }^{\circledR} 19 \%$ v/v was slightly but significantly better than with FM $85^{\circledR}$.

Key-words: nutrition; bone mineralization; growth; infant, newborn; infant, premature.

\section{Introdução}

As taxas de sobrevivência e a qualidade de vida de recémnascidos de muito baixo peso (RNMBP), ou seja, aqueles que possuem peso ao nascer menor que $1500 \mathrm{~g}$, tem melhorado significativamente nas últimas décadas. Isso se deve aos progressos técnico-científicos incorporados na assistência dessas crianças, cujo bom desenvolvimento também está ligado ao processo nutricional pós-natal imediato, período de rápido crescimento e maturação estrutural que exige aporte adequado de nutrientes em quantidade e qualidade para garantir uma boa evolução pôndero-estatural, sem iatrogenias carenciais ou sobrecargas metabólico-digestivas indesejáveis ${ }^{(1)}$. Assim, após o nascimento, procura-se oferecer precocemente a essas crianças aporte adequado de proteínas, energia, vitaminas e sais minerais no intuito de manter seu crescimento o mais próximo possível do esperado no meio intrauterino ${ }^{(2-4)}$.

Estudos recentes indicam que o leite humano $(\mathrm{LH})$ pode ser melhor do que as fórmulas especiais para prematuros, tanto para o crescimento imediato quanto para o desenvolvimento em longo prazo ${ }^{(5,6)}$, além de diminuir a incidência de infecções no período neonatal e acelerar a maturação gastrintestinal dessas crianças ${ }^{(7)}$. Contudo, alguns estudos demonstraram que o $\mathrm{LH}$, mesmo o da própria mãe do prematuro, sem adições, não fornece quantidade adequada de diversos nutrientes, dentre eles proteínas e minerais, em especial cálcio e fósforo ${ }^{(7-9)}$, promovendo crescimento insatisfatório e desmineralização óssea em RNMBP não-suplementados, o que caracteriza a doença óssea metabólica ou osteopenia da prematuridade ${ }^{(9-11)}$. A doença metabólica óssea, que atingia cerca de $30 \%$ dos prematuros com peso até $1500 \mathrm{~g}$ ao nascer, com fraturas em $24 \%$ delas, era diagnosticada entre seis e 12 semanas de vida pós-natal ${ }^{(4,12,13)}$, mostrando níveis séricos de fósforo baixos, fosfatase alcalina aumentada, cálcio e magnésio geralmente normais e taxas de reabsorção de fósforo urinário elevadas $^{(8)}$, além da hipercalciúria e níveis altos de 1,25(OH) D-calciferol, metabólito ativo da vitamina $\mathrm{D}$, que aumenta a absorção intestinal de cálcio e a liberação de cálcio e fósforo para o soro ${ }^{(4,11)}$. Porém, quando o aporte de fósforo é insuficiente, como ocorre ao se usar exclusivamente $\mathrm{LH}$ para alimentar bebês prematuros, surge a hipofosfatemia, que limita a quantidade de cálcio que se deposita nos ossos, aumenta a calcemia e promove hipercalciúria. Essa última vem sendo proposta como um eficiente marcador da doença óssea metabólica do prematuro ${ }^{(13,14)}$.

A Academia Americana de Pediatria recomenda que as fórmulas para neonatos pré-termo contenham 140 a $160 \mathrm{mg}$ de cálcio/100kcal $(220-250 \mathrm{mg} / \mathrm{kg} / \mathrm{dia}), 95$ a $108 \mathrm{mg}$ de fósforo/100kcal (150-170mg/kg/dia) e 400UI de vitamina D. A Sociedade Europeia de Gastroenterologia e Nutrição (ESPGAN) recomenda 70 a 140mg de cálcio/100kcal e 50 a $90 \mathrm{mg}$ de fósforo/ $100 \mathrm{kcal}^{(9,12,13,15)}$. É desejável, pois, que se disponha de dieta bem balanceada, com proteínas da própria espécie, teor calórico suficiente, sais minerais em quantidades e proporções adequadas e osmolaridade apropriada ${ }^{(14,16,17)}$ para bem nutrir os RNMBP. Essa dieta pode ser obtida com LH acrescido de "complementos" ou "aditivos" especiais, que se tornaram disponíveis no Brasil nos últimos cinco anos, ou de sua mistura, em proporções variadas, com fórmula especial para prematuros em concentrações mais elevadas que as usuais sem, no entanto, exceder a osmolaridade tolerada por essas crianças, o que já era utilizado nos Berçários do Hospital das Clínicas da Faculdade de Medicina de Ribeirão Preto da Universidade de São Paulo (HCFMRP-USP) há vários anos, com diminuição significativa da osteopenia em $\operatorname{RNMBP}^{(3,15,18)}$.

Nesse contexto, o objetivo do estudo foi comparar o efeito de dois complementos para o leite humano na nutrição de recém-nascidos pré-termo de muito baixo peso, enfatizando-se o crescimento, o metabolismo ósseo e a sua tolerabilidade ${ }^{(7)}$.

\section{Métodos}

Este estudo prospectivo foi iniciado com 25 neonatos prematuros de ambos os sexos e concluído com 22 (11 em cada grupo). Foram incluídos todos os pacientes com peso de nascimento até $1500 \mathrm{~g}$, admitidos nos berçários do HCFMRP-USP no período de janeiro de 2000 a outubro de 2001, que se encontravam em nutrição enteral exclusiva e clinicamente estáveis com 15 dias de vida e cujos pais assinaram o Termo de Consentimento Livre e Esclarecido, como estabelecido pelas normas éticas vigentes para estudos em seres humanos. O projeto foi aprovado pelo Comitê de Ética em Pesquisa do HCFMRP-USP.

Os recém-nascidos tiveram suas idades gestacionais calculadas pela regra de Nägelle, baseada na data de início do último 
ciclo menstrual da mãe, sendo confirmadas por avaliações somato-neurológicas realizadas pela autora, conforme proposto por Ballard et a ${ }^{(19)}$, e foram classificados quanto ao crescimento intraútero segundo Alexander ${ }^{(20)}$, com acompanhamento do $15^{\circ}$ dia de vida até a alta, que ocorria quando a criança atingia $2000 \pm 20$ g. Não foram incluídos neonatos portadores de malformações congênitas e/ou patologias que pudessem interferir no crescimento e no desenvolvimento da criança, nem gemelares.

Foram realizadas avaliações das crianças aos $15 \pm 2$ dias de vida, a cada 15 dias e na alta, aferindo-se peso, comprimento, perímetro cefálico e prega cutânea tricipital média esquerda. Dosaram-se: cálcio, fósforo, magnésio e creatinina no soro e na urina de seis horas e fosfatase alcalina no soro a cada 15 dias e na alta hospitalar. Foram calculadas as taxas de reabsorção tubular de fósforo no início e no final do estudo, quando também foram realizadas radiografias de punho esquerdo para avaliação da mineralização óssea.

Os pacientes foram distribuídos pareadamente, por sorteio, em dois grupos: Grupo A, formado por 11 prematuros, que receberam $\mathrm{LH}$ enriquecido com o "fortificador" FM $85^{\circledR}$ na proporção de $5 \mathrm{~g}$ para cada $100 \mathrm{~mL}$ de leite humano; Grupo B, com 11 neonatos prematuros que receberam LH adicionado, volume a volume, com fórmula especial para prematuros $\left(\operatorname{PreNan}^{\circledR}\right)$ na concentração de $19 \%$ (19g de pó e água q.s.p. $100 \mathrm{~mL}$ ).

Todos os neonatos receberam leite humano da própria mãe sem adições até o $15^{\circ}$ dia de vida e, posteriormente, suas respectivas dietas, no volume total de $160 \mathrm{~mL} / \mathrm{kg} / \mathrm{dia}$, até a alta, que acontecia quando a criança atingia $2000 \pm 20 \mathrm{~g}$.
Quando o leite da própria mãe não era suficiente, utilizava-se leite humano de banco de leite. Todas as crianças receberam suplementação vitamínica a partir do $8^{\circ}$ dia de vida e de ferro a partir do $16^{\circ}$ dia, de acordo com a rotina já estabelecida do berçário do HCFMRP-USP.

A Tabela 1 apresenta a composição média de nutrientes de leite humano (LH) puro, acrescido de $5 \mathrm{~g}$ de aditivo $\mathrm{FM} 85^{\circledR}$ ou de fórmula especial para prematuros (FEPT) a $19 \%$, bem como as osmolaridades e relações $\mathrm{Ca} / \mathrm{P}$ resultantes das adições. As dietas eram enviadas ao berçário em recipientes similares, apenas com rótulo para o usuário. As duas formulações tinham aspecto semelhante, não permitindo que se identificasse qual dieta estava sendo oferecida à criança, e os enfermeiros eram orientados a agitarem os frascos com as dietas antes de sua administração.

A tolerabilidade digestiva foi avaliada através da comparação e verificação de: episódios de distensão abdominal; resíduos gástricos (quando em sonda gástrica) ou vômitos; presença ou suspeita de casos de enterocolite necrosante.

Utilizou-se o teste $t$ de Student para comparar as médias dos valores obtidos nas diferentes idades nos dois grupos, com cálculos efetuados pelo programa Epi-info. O teste do qui-quadrado foi aplicado para comparar a tolerabilidade. Considerou-se significativo $p<0,05$.

\section{Resultados}

A Tabela 2 apresenta a médias e o respectivo desvio padrão da idade gestacional, peso e comprimento de nascimento das

Tabela 1 - Composição média, por 100mL, de nutrientes de leite humano (LH) puro e das fórmulas oferecidas durante o estudo

\begin{tabular}{|c|c|c|c|}
\hline & Leite humano (LH) & $\begin{array}{c}\mathrm{LH}(100 \mathrm{~mL})+ \\
5 \mathrm{~g} \mathrm{FM} 85^{\circledR}\end{array}$ & $\begin{array}{c}\text { LH }(50 \mathrm{~mL})+ \\
\text { FEPT } 19 \%(50 \mathrm{~mL})\end{array}$ \\
\hline Proteína $(\mathrm{g})$ & 1,2 & 1,9 & 2 \\
\hline Gordura (g) & 3,8 & 3,7 & 4,2 \\
\hline Carboidratos $(\mathrm{g})$ & 7 & 10,2 & 8,8 \\
\hline Energia (kcal) & 67 & 81,7 & 80,2 \\
\hline Sódio (mEq) & 0,7 & 1,8 & 1,1 \\
\hline Potássio (mEq) & 1,3 & 1,6 & 2 \\
\hline Cálcio (mg) & 31 & 78,8 & 62,2 \\
\hline Fósforo (mg) & 15 & 47,1 & 37,8 \\
\hline Cloreto (mEq) & 1,2 & 1,7 & 1,4 \\
\hline Magnésio (mg) & 3,7 & 5,5 & 7 \\
\hline Osmolaridade (mmol/L) & 278 & 391 & 378 \\
\hline Relação cálcio:fósforo & $2: 01$ & $1,7: 1$ & $1,7: 1$ \\
\hline Densidade calórica (kcal/100mL) & 67 & 81,7 & 80,2 \\
\hline
\end{tabular}

FEPT: fórmula especial para prematuros 
Tabela 2 - Idade gestacional, peso e comprimento ao nascer e classificação quanto ao crescimento intraútero

\begin{tabular}{lccc}
\hline & Grupo A & Grupo B & Valor de $\boldsymbol{p}$ \\
\cline { 2 - 3 } & $\mathbf{( n = 1 1 )}$ & $\mathbf{( n = 1 1 )}$ & 0,42 \\
\hline Idade gestacional (semanas) & $33 \pm 2$ & $33 \pm 2$ & 0,70 \\
Peso (g) & $1295 \pm 185$ & $1317 \pm 185$ & 0,70 \\
Comprimento (cm) & $36,5 \pm 1,8$ & $36,1 \pm 1,8$ & 1,00 \\
PIG/AIG & $10-1$ & $10-1$ & \\
\hline
\end{tabular}

PIG: pequeno para idade gestacional; AIG: grande para a idade gestacional.

Tabela 3 - Média e desvio-padrão do peso, comprimento, perímetro cefálico e prega cutânea tricipital média esquerda dos RNMBP dos dois grupos no início e ao final da observação

\begin{tabular}{lcccccc}
\hline & \multicolumn{3}{c}{ Início } & \multicolumn{3}{c}{ Final } \\
\cline { 2 - 7 } & $\begin{array}{c}\text { Grupo A } \\
(\mathbf{n = 1 1 )}\end{array}$ & $\begin{array}{c}\text { Grupo B } \\
(\mathbf{n = 1 1 )}\end{array}$ & Valor de $\boldsymbol{p}$ & $\begin{array}{c}\text { Grupo A } \\
(\mathbf{n = 1 1 )}\end{array}$ & $\begin{array}{c}\text { Grupo B } \\
(\mathbf{n = 1 1 )}\end{array}$ & Valor de $\boldsymbol{p}$ \\
\hline Peso $(\mathrm{g})$ & $1.380 \pm 251$ & $1.373 \pm 136$ & 0,90 & $2.017 \pm 28$ & $2.014 \pm 30$ & 0,80 \\
Comprimento $(\mathrm{cm})$ & $40,0 \pm 2$ & $39,8 \pm 1,6$ & 0,80 & $43,1 \pm 1,1$ & $43,3 \pm 1,1$ & 0,68 \\
Perímetro cefálico $(\mathrm{cm})$ & $28,4 \pm 1,8$ & $29,5 \pm 1,9$ & 0,19 & $30,8 \pm 2,6$ & $32,1 \pm 0,9$ & 0,16 \\
Prega cutânea tricipital $(\mathrm{mm})$ & $2,11 \pm 0,51$ & $1,90 \pm 0,33$ & 0,26 & $2,97 \pm 0,57$ & $2,37 \pm 0,36$ & 0,01 \\
\hline
\end{tabular}

Tabela 4 - Média \pm desvio-padrão do ganho ponderal diário (g/dia); velocidade de ganho ponderal (g/kg/dia); tempo de permanência no estudo (dias); tempo de internação (dias) do RNMBP dos grupos A e B

\begin{tabular}{lccc}
\hline & Grupo A & Grupo B & \multirow{2}{*}{ Valor de $\boldsymbol{p}$} \\
\cline { 2 - 3 } & $\mathbf{( n = 1 1 )}$ & $\mathbf{( n = 1 1 )}$ & 0,03 \\
Ganho ponderal diário (g/dia) & $23,8 \pm 5,3$ & $29,8 \pm 6,4$ & 0,03 \\
Velocidade de ganho ponderal (g/kg/dia) & $15,5 \pm 2,4$ & $17,5 \pm 3,6$ & 0,56 \\
Tempo de permanência no estudo (dias) & $25,0 \pm 11,0$ & $22,8 \pm 7,2$ & 0,57 \\
Tempo de internação (dias) & $40,3 \pm 11,6$ & $37,9 \pm 7,5$ & \\
\hline
\end{tabular}

crianças incluídas nos dois grupos, não havendo diferença entre ambos, assim como na proporção de bebês pequenos e grandes para a idade gestacional (PIG/AIG), evidenciando que os grupos eram comparáveis.

A Tabela 3 demonstra os valores médios e respectivos desvios-padrão do peso, comprimento e perímetro cefálico das crianças dos dois grupos no início e ao final da observação, sem diferenças entre ambos.

Quanto às pregas cutâneas tricipitais médias, notou-se que, no início, eram semelhantes nos dois grupos, mas, no final do estudo, eram maiores nos RNMBP do Grupo A $(p<0,01)$, sugerindo que os neonatos desse grupo possuíam maior panículo adiposo.

A Tabela 4 apresenta as médias e desvios-padrão do ganho ponderal diário, da velocidade de ganho ponderal e do tempo de internação dos RNMBP dos dois grupos, observando-se que os do Grupo B apresentaram ganho de peso médio diário maior $(p<0,05)$, ou seja, precisaram de menor tempo para atingir o peso de alta, sem, contudo, atingir significância estatística, apenas alcançada pela velocidade de ganho ponderal $(p<0,01)$.

A Tabela 5 mostra que os níveis séricos médios de cálcio, fósforo e magnésio iniciais e finais das crianças dos dois grupos estavam dentro da faixa da normalidade para a idade e não diferiam estatisticamente. Já os níveis de fosfatase alcalina eram elevados nos dois grupos no início $(p>0,05)$ e decaíram até o final, tendo as crianças do Grupo B ( $\mathrm{LH}+\mathrm{FEPT}-\mathrm{v} / \mathrm{v})$ apresentado níveis mais baixos $(p<0,05)$.

A excreção urinária de cálcio foi mais elevada nas crianças do Grupo A em relação às do Grupo B nas duas épocas estudadas, como apresentado na Tabela 6, porém sem significância estatística. $\mathrm{O}$ inverso ocorreu com o fósforo, cuja excreção era mínima no início em ambos os grupos e, ao final, continuou mínima no Grupo A, mas aumentou significativamente no Grupo B $(p<0,05)$. A excreção de magnésio foi similar em ambos os grupos tanto no início quanto no final da observação. Quanto às taxas de reabsorção tubular de fósforo (\%TPR $)^{(21)}$, verificou-se que eram muito elevadas 
Tabela 5 - Médias \pm desvio-padrão dos níveis séricos iniciais e finais de cálcio, fósforo, magnésio e fosfatase alcalina dos 11 RNMBP dos Grupos A e B

\begin{tabular}{lccccccc}
\hline & \multicolumn{3}{c}{ Inicial } & & \multicolumn{3}{c}{ Final } \\
\cline { 2 - 3 } & Grupo A & Grupo B & \multirow{2}{*}{ Valor de $\boldsymbol{p}$} & Grupo A & Grupo B & \multirow{2}{*}{ Valor de $\boldsymbol{p}$} \\
\cline { 2 - 3 } & $\mathbf{( n = 1 1 )}$ & $\mathbf{( n = 1 1 )}$ & & $\mathbf{( n = 1 1 )}$ & $\mathbf{( n = 1 1 )}$ & \\
\hline Cálcio $(\mathrm{mg} / \mathrm{dL})$ & $10,0 \pm 1,2$ & $10,2 \pm 1,7$ & 0,76 & $9,9 \pm 1,8$ & $9,8 \pm 2,1$ & 0,9 \\
Fósforo $(\mathrm{mg} / \mathrm{dL})$ & $5,3 \pm 1,2$ & $5,1 \pm 1,0$ & 0,73 & $5,6 \pm 1,9$ & $7,0 \pm 1,9$ & 0,1 \\
Magnésio $(\mathrm{mg} / \mathrm{dL})$ & $1,7 \pm 0,3$ & $1,9 \pm 0,3$ & 0,75 & $1,8 \pm 0,4$ & $1,8 \pm 0,3$ & 0,8 \\
Fosfatase alcalina $(\mathrm{Ul} / \mathrm{dL})$ & $342 \pm 83$ & $296 \pm 126$ & 0,33 & $315 \pm 87$ & $232 \pm 95$ & 0,04 \\
\hline
\end{tabular}

Tabela 6 - Média \pm desvio-padrão da excreção urinária de cálcio, fósforo e magnésio e taxas de reabsorção tubular de fósforo (\%TRP) nos RNMBP dos dois grupos no início e no final do estudo

\begin{tabular}{|c|c|c|c|c|c|c|}
\hline & \multicolumn{3}{|c|}{ Inicial } & \multicolumn{3}{|c|}{ Final } \\
\hline & Grupo A & Grupo B & & Grupo A & Grupo B & \\
\hline & $(n=11)$ & $(n=11)$ & Valor de $p$ & $(n=11)$ & $(n=11)$ & Valor de $p$ \\
\hline Cálcio (mg/dL) & $9,6 \pm 4,1$ & $5,9 \pm 5,3$ & 0,06 & $10,3 \pm 11,2$ & $6,7 \pm 5,3$ & 0,33 \\
\hline Fósforo (mg/dL) & $1,9 \pm 1,0$ & $4,1 \pm 2,7$ & 0,02 & $3,8 \pm 7,2$ & $15,9 \pm 17,6$ & 0,04 \\
\hline Magnésio (mg/dL) & $0,7 \pm 0,5$ & $0,6 \pm 0,2$ & 0,68 & $0,9 \pm 0,4$ & $0,6 \pm 0,2$ & 0,03 \\
\hline \%TRP & $98,4 \pm 0,8$ & $93,1 \pm 14,2$ & 0,23 & $93,5 \pm 15,3$ & $86,7 \pm 17,2$ & 0,34 \\
\hline
\end{tabular}

em ambos os grupos no início e diminuíram no final da observação mais acentuadamente no Grupo B, porém sem diferença significativa entre os grupos (Tabela 6).

A tolerabilidade das dietas não apresentou diferença entre os dois grupos, apesar de três crianças selecionadas para o Grupo A terem sido excluídas por suspeita nãoconfirmada de enterocolite necrosante.

A avaliação da mineralização óssea, com técnica radiográfica e leitura padronizada, não evidenciou diferenças entre os grupos, nem variações intragrupo do início para o final do estudo, pois nenhum exame evidenciou sinais de desmineralização óssea.

\section{Discussão}

O nascimento prematuro implica a perda de importante período de desenvolvimento intraútero do feto, quando há grande incremento de cálcio e fósforo em seu organismo, com intensa mineralização óssea e rápido crescimento somático $^{(3,6,13)}$. Após o nascimento, esses recém-nascidos devem receber uma alimentação que supra as necessidades nutricionais diárias e não provoque sobrecarga em seu sistema digestivo ainda imaturo(22). Apesar dos contínuos avanços na nutrição de prematuros obtidos nos últimos anos, a alimentação ideal para esse importante contingente de crianças continua a ser objeto de muitos estudos ${ }^{(1,8)}$.

Assume-se, atualmente, que a alimentação enteral dos recém-nascidos pré-termo deva ser iniciada o mais preco- cemente possível, com leite humano, preferencialmente de suas próprias mães, recomendando-se fórmula especial para prematuros se não houver disponibilidade de $\operatorname{LH}^{(9,21,22)}$. Contudo, quando se atinge o volume enteral total, o LH deve ser suplementado, a fim de fornecer adequado aporte de nutrientes ${ }^{(23,24)}$, pois mesmo o $\mathrm{LH}$ da própria mãe do prematuro não supre todas as suas necessidades e pode não proporcionar ganho ponderal adequado ${ }^{(7,9,24)}$.

Assim, o presente estudo objetivou comparar a eficácia de duas formas de suplementar o LH quanto ao ganho ponderal, tempo de hospitalização, metabolismo ósseo e sua mineralização: fórmula especial para prematuro em concentração não usual (19\%) ou “aditivo” especial, comercialmente disponível no Brasil na época dessa investigação (FM $85^{\circledR}$ ). Os dois grupos mostraram-se comparáveis ao nascer e também no início da observação, com idade gestacional semelhante, além de peso, comprimento e perímetro cefálico muito próximos. $\mathrm{O}$ ganho ponderal proporcionado pelas duas dietas, de acordo com a literatura recente, pode ser considerado satisfatório, pois está em torno de 25 a 30g/dia (Tabela 4), sendo o ganho ponderal diário significativamente maior no Grupo B (29,7g/ dia) do que no A (23,8g/dia), sugerindo maior eficácia do LH+FEPT 19\%, v/v. O melhor ganho de peso do Grupo $\mathrm{B}$ resultou em menor tempo médio de hospitalização das crianças deste grupo, em média 2,4 dias, sem significância estatística, mas com importância econômica e sociofamiliar. A alta mais precoce resultou em menor custo hospitalar e inserção mais rápida da criança no seio familiar ${ }^{(25)}$. O aumento 
de comprimento e do perímetro cefálico das crianças dos dois grupos foram também satisfatórios e não houve diferenças entre ambos, embora as crianças do Grupo B tenham apresentado incrementos ligeiramente maiores, como mostra a Tabela 3.

Quanto às pregas cutâneas, utilizadas para estimar a gordura subcutânea e, por extensão, a gordura corporal, pôde-se verificar que as crianças do Grupo A, apesar de apresentarem menor velocidade de ganho ponderal ( $\mathrm{g} / \mathrm{kg} / \mathrm{dia})$, mostraram panículo adiposo significativamente mais desenvolvido do que aquelas do Grupo B (Grupo A=2,97 mm versus Grupo $\mathrm{B}=2,37 \mathrm{~mm}, p<0,05$ ), o que pode ser consequência dos nutrientes das dietas, situando-se entre os percentis 75 e 50 , respectivamente, de recém-nascidos adequados para a idade gestacional com peso equivalente ${ }^{(7)}$. Questiona-se, ainda, o que é melhor nessa fase da vida: ter mais ou menos gordura corporal. Esse debate abrange as repercussões das dietas de prematuros sobre alterações cardiovasculares (aterosclerose precoce), relatadas em adultos jovens ex-prematuros ${ }^{(26)}$. É preciso, pois, definir a dieta ideal para essas crianças, que proporcione um bom ganho ponderal sem sobrecarga digestiva e metabólica aguda e sem efeitos deletérios em longo prazo.

No presente estudo, ofereceu-se às crianças estudadas 100 e $126 \mathrm{mg} / \mathrm{kg} / \mathrm{dia}$ de cálcio e 60 e $75 \mathrm{mg} / \mathrm{kg} / \mathrm{dia}$ de fósforo, respectivamente para os Grupos A e B, quantidades consideradas suficientes e em proporção adequada $(1,7: 1)$ para a boa absorção desses minerais pelo intestino. Em decorrência disso, esperava-se uma adequada mineralização óssea em ambos os grupos, com níveis séricos e urinários de cálcio e fósforo normais e taxas de reabsorção tubular de fósforo altas, porém normais para idade, o que foi confirmado. Os níveis urinários de cálcio e fósforo apresentaram grande variabilidade, porém sem diferenças significativas entre os grupos, sendo que as crianças do Grupo A excretarem mais cálcio pela urina que as do Grupo $B$, sugerindo menor adequação da dieta do Grupo A quanto ao aporte de cálcio e fósforo. A sugestão de menor adequação da dieta do Grupo A é reforçada pelo achado de menor excreção de fósforo e maior \% TPR final neste grupo ${ }^{(27)}$.

Os níveis de fosfatase alcalina, ligeiramente elevados (@300UI/dL) em relação aos de adultos no início do estudo, eram normais para a faixa etária e não diferiam entre os grupos. Ao final do estudo, os níveis de fosfatase alcalina das crianças de ambos os grupos eram menores do que os iniciais, sendo os valores médios do Grupo B mais baixos do que os do Grupo
$\mathrm{A}$, indicando maior atividade osteoblástica nas crianças deste grupo, o que sugere maior remodelação óssea ou solubilização de hidroxiapatita devido à menor absorção de fósforo e cálcio da dieta $\mathrm{A}\left(\mathrm{LH}+\mathrm{FM} 85^{\oplus}\right)$, lembrando-se que esta dieta oferece $25 \%$ mais cálcio e fósforo que a dieta B. Uma possível explicação para este achado seria a menor absorção desses minerais devido à menor solubilidade do aditivo então disponível e de seus sais de cálcio e fósforo, com consequente menor biodisponibilidade destes micronutrientes no $\mathrm{LH}+\mathrm{FM} 85^{\circledR}$ em relação ao $\mathrm{LH}$ adicionado com fórmula para prematuros ${ }^{(3,4)}$. Deve-se ainda ressaltar que o aditivo $\mathrm{FM} 85^{\circledR}$ recebeu modificações em sua formulação em relação ao produto oferecido neste estudo, obviamente para aprimorá-lo, pois outros autores verificaram que sua adição ao LH não supria as necessidades de minerais de prematuros extremos ${ }^{(4,23,27)}$.

Quanto ao magnésio, não houve diferença significativa nos níveis séricos nos dois grupos, o que indica adequado aporte de magnésio nas duas dietas, resultado relatado unanimemente pela literatura ${ }^{(6)}$.

No presente estudo, a mineralização óssea das crianças dos dois grupos, com a tecnologia usada (raio X simples de punhos), foi considerada satisfatória, pois nenhuma criança apresentou sequer rarefação óssea, grau mínimo de osteopenia. Isso demonstra que, nesse aspecto, ambas as dietas podem ser consideradas adequadas. Contudo, as dosagens séricas de fósforo e fosfatase alcalina e as taxas de reabsorção tubular de fósforo (\%TPR) no final do estudo sugeriram que a dieta do Grupo B, em termos de mineralização óssea, foi mais adequada, pois os níveis de fósforo eram mais elevados e os de fosfatase alcalina e de TPR, mais baixos. Isso poderia ser confirmado, ou não, por tecnologia mais sensível, como a densitometria óssea ${ }^{(7)}$, que só recentemente se tornou disponível no serviço.

Pode-se concluir que ambas as dietas foram bem toleradas e proporcionaram adequado crescimento e mineralização óssea dos RNMBP. Contudo, recomendam-se estudos mais amplos e sofisticados (densitometria óssea e análise da composição corporal pelo DEXA e/ou estudos com radioisótopos estáveis ${ }^{(28)}$ e com maior número de pacientes, incluindo prematuros extremos $(<1000 \mathrm{~g})$, com a nova formulação do FM $85^{\circledR}$ para que se possa comparar a deposição de gordura no tecido subcutâneo, a biodisponibilidade de fósforo e cálcio e a mineralização óssea em relação à promovida pelo leite humano adicionado volume a volume com fórmula especial para prematuro. 


\section{Referências bibliográficas}

1. Abdallah VO. Avaliação do crescimento e estudo do cálcio, fósforo e magnésio em recém-nascidos de muito baixo peso ao nascer durante os primeiros dois meses de vida [tese de mestrado]. Ribeirão Preto (SP): USP; 1989.

2. Chan DK. Enteral nutrition of the very low birth weight (VLBW) infant. Ann Acad Med Singapore 2001;30:174-82.

3. Demarini S. Calcium and phosphorus nutrition in preterm infants. Acta Paediatr (Suppl) 2005;94:87-92.

4. Rigo J, Pieltain C, Salle B, Senterre J. Enteral calcium, phosphate and vitamin $\mathrm{D}$ requirements and bone mineralization in preterm infants. Acta Paediatr 2007;96:969-74.

5. Atkinson SA. Human milk feeding of the micropremie. Clin Perinatol 2000;27:235-47.

6. Trindade CE. Minerals in the nutrition of extremely low birth weight infants. J Pediatr (Rio J) 2005;81:S43-51.

7. Kuschel CA, Harding JE. Multicomponent fortified human milk for promoting growth preterm infants. Cochrane Database Syst Rev 2004:CD000343.

8. Abdallah VO. Estudo do crescimento pondero-estatural, do metabolismo protéico e do estado relativo ao cálcio, fósforo e magnésio em crianças com muito baixo peso $(\leq 1500 \mathrm{~g})$ e alimentadas com três diferentes dietas do $15^{\circ}$ ao $45^{\circ}$ dias de vida [tese de doutorado]. Ribeirão Preto (SP): USP; 1993.

9. Greer FR. Feeding the premature infant in the $20^{\text {th }}$ century. J Nutr 2001;131:426S-30.

10. Eliakim A, Nemet D. Osteopenia of prematurity - the role of exercise in prevention and treatment. Pediatr Endocrinol Rev 2005;2:675-82.

11. Harrison CM, Johnson K, McKechnie E. Osteopenia of prematurity: a national survey and review of practice. Acta Paediatr 2008;97:407-13.

12. Demarini S, Tsang RC. Calcium and phosphorus nutrition in infants. J Pediatr Gastroenterol Nutr 1995;21:367-73.

13. Catache M, Leone CR. Critical analysis of pathophysiological, diagnostic and therapeutic aspects of metabolic bone disease in very low birth weight infants. J Pediatr (Rio J) 2001;77(Suppl 1):S53-62.

14. Catache M, Leone CR. Role of plasma and urinary calcium and phosphorus measurements in early detection of phosphorus deficiency in very low birthweight infants. Acta Paediatr 2003;92:76-80.
15. American Academy of Pediatrics. Committee on Nutrition. Nutritional needs of low-birth-weigth infants. Pediatrics 1985;75:976-86.

16. Schanler RJ. The use of human milk for premature infants. Pediatr Clin North Am 2001;48:207-19.

17. Koo WW, Gupta JM, Nayanar VV, Wilkinson M, Posen S. Skeletal changes in preterm infants. Arch Dis Child 1982;57:447-52.

18. Rigo J, De Curtis M, Pieltain C, Picaud JC, Salle BL, Senterre J. Bone mineral metabolism in the micropremie. Clin Perinatol 2000;27:147-70.

19. Ballard JL, Novak KK, Driver M. A simplified score for assessment of fetal maturation of newly born infants. J Pediatr 1979;95:769-74.

20. Alexander GR, Himes JH, Kaufman RB, Mor J, Kogan M. A United States national reference of fetal growth. Obstet Gynecol 1996;87:63-8.

21. Tobias GJ, McLaughlin RF Jr, Hopper J Jr. Endogenous creatinine clearance. A valuable clinical test of glomerular filtration and a prognostic guide in chronic renal disease. N Engl J Med 1962;266:317-23.

22. Schanler RJ. The use of human milk for premature infants. Pediatr Clin North Am 2001;48:207-19.

23. Loui A, Raab A, Obladen M, Brätter P. Calcium, phosphorus and magnesium balance: FM 85 fortification of human milk does not meet mineral needs of extremely low birthweight infants. Euro J Clin Nutr 2002;56: 228-35.

24. Kuschel CA, Harding JE. Protein supplementation of human milk for promoting growth in preterm infants. Cochrane Database Syst Rev 2000: CD000433.

25. Borges EG. Uso de parâmetros antropométricos e bioquímicos na avaliação do estado nutricional de recém-nascidos de termo e pré-termo [tese de doutorado]. Ribeirão Preto (SP): USP; 1990

26. Singhal A, Lucas A. Early origins of cardiovascular disease: is there a unifying hypothesis? Lancet 2004;363:1642-5.

27. Pohlandt F, Mihatsch WA. Reference values for urinary calcium and phosphorus to prevent osteopenia of prematurity. Pediatr Nephrol 2004;19: 1192-3.

28. Greer FR. Post-discharge nutrition: what does the evidence support? Semin Perinatol 2007;31:89-95. 